\title{
INCORPORATING WAITING TIME IN COMPETITIVE LOCATION MODELS: FORMULATIONS AND HEURISTICS ${ }^{1}$
}

\author{
Francisco Silva $^{a}$, Daniel Serrab \\ ${ }^{\text {a }}$ GREL, IET, Universitat Pompeu Fabra, Ramon Trias Fargas, 25-27, 08005 Barcelona, Spain. \\ CEEAplA, Universidade dos Açores, Rua da Mae de Deus, 9502 Ponta Delgada, Portugal \\ email: francisco.silva@upf.edu

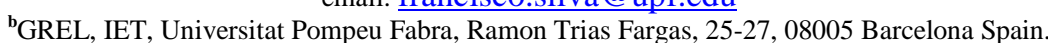 \\ email: daniel.serra@upf.edu
}

\begin{abstract}
In this paper we propose a metaheuristic to solve a new version of the Maximum Capture Problem. In the original MCP, market capture is obtained by lower traveling distances or lower traveling time, in this new version not only the traveling time but also the waiting time will affect the market share. This problem is hard to solve using standard optimization techniques. Metaheuristics are shown to offer accurate results within acceptable computing times.
\end{abstract}

Keywords: Market capture, queuing, ant colony optimization.

JEL:C61,L80.

\footnotetext{
${ }^{1}$ This research has been possible thanks to the grant SFRH/BD/2916/2000 from the Ministerio da Ciência e da Tecnologia, Fundação para a Ciência e a Tecnologia of the Portuguese government.
} 


\section{Introduction.}

ReVelle's Maximum Capture Problem (1986) initiated a series of studies on the location of retail facilities in discrete space (see Serra and ReVelle (1995)). The MAXCAP model makes the following assumptions: (1) the product sold is homogeneous, (2) the consumer's decision on patronizing the store is based on distance and (3) unit costs are the same in all stores regardless of ownership. Examples of services that best fit these three assumptions can be found mainly in the fast food sector, in convenience stores and in the banking sector. However, in all these examples, not only the distance but also waiting time seems to determine the consumer's decision. The number of persons the consumer finds in queue, when he or she arrives at the store, can be a measure for the consumer's perception of waiting time. Furthermore, the waiting time for one visit may affect future decisions as to which store to patronize the next visit. This seems to be quite relevant for some retail stores, fast food restaurants or ATM machines.

Kohlberg (1983), in pioneer work in the same line of research, considers a variant of the classical Hotelling model for store locations. The author assumes that when choosing a store, consumers take into account not only travel time but also waiting time for the service at each store, which in turn depends on the number of consumers patronizing that store. Assuming that each consumer makes the decision that minimizes travel time plus waiting time, stores' market shares are shown to be continuous functions of their locations.

There is also a general consensus that the distances may be interpreted in a functional, proximity, or similarity context rather than in a geometrical one. Our claim is that in some types of services, waiting time has a strong impact on the consumer's perception of proximity.

In chapter 1 we will revise some literature on competitive spatial modeling. In chapter 2 we describe a model, which incorporates explicitly waiting time, and in chapter 3 we propose a metaheuristic to solve the model. Some results of our computational experiments are described in chapters 4 and 5 . 


\section{Literature Review.}

In its simplest scenario the game works as follows: the leader firm locates a number of facilities, anticipating that the follower will react to the location pattern. The follower, in turn, will then solve the conditional location problem of locating his own facilities given the leader's chosen locations. Following Hakimi (1983), we refer to the leader's problem of locating a fixed number of facilities, knowing that the follower will subsequently locate his own facilities, as an (r|p) centroid problem. The follower, in turn, will then face a location pattern of the facilities of the leader and, given that, optimize the location of his own facilities. This is known as the (r|Xp) medianoid problem.

A typical model in the former category is the MaxCap (maximum capture) model introduced by ReVelle (1986). The model formulated by ReVelle finds the optimal location on a network considering that each demand point will patronize the closest facility. Several authors have expanded ReVelle's formulation: Eiselt and Laporte (1989) generalize ReVelle's findings in two directions: they allow differential weights for the facilities and they leave a parameter of the cost function variable so as to facilitate sensitivity analysis, Serra and ReVelle (1993) introduce in the model facilities that are hierarchical in nature and where there is competition at each level of the hierarchy, the same authors, Serra and ReVelle (1994), account the possible reaction from competitors to the entering firm in the preemptive location problem, in which the leader wishes to preempt the entering firm in its bid to capture market share to the maximum extent possible. Serra, Ratick and ReVelle (1996) offer a modification of the MaxCap problem in which they consider uncertainty. The authors consider different future scenarios with respect to demand and/or the location of competitors.

Most competitive location problems were at first developed under the hypothesis that different firms provide the same indistinguishable product and that all customers have the same preferences, i.e., the same deterministic utility function. Some literature refers to the topic of dropping the hypothesis of the homogeneity of the product. 
In Drezner (1994), customers base facility choice on a utility function that incorporates a facility's attributes and the distance to the facility. Although customers are no longer assumed to patronize the closest facility, customers at a certain demand point apply the same utility function.

Drezner and Drezner (1996) assume the utility function to change from one consumer to another for customers located at the same demand point. Using this assumption the "all or nothing" property disappears.

Serra, Eiselt, Laporte and ReVelle (1999) developed two models allowing different customer choice rules. One model assumes that customers consider the closest facility of each firm and then patronize the two facilities in proportion to the customer-facility distance. The other model assumes that the demand captured by a facility is affected by the existence and location of all facilities of both firms.

Other improvements over the initial maximum capture model refer to minimum market shares that firms need to capture in order to survive. Carreras and Serra (1998) present a model that locates the maximum number of services that can coexist in a given region without having losses, taking into account that they need a minimum demand level in order to survive.

Serra, ReVelle and Rosing (1999) considered the problem of locating several facilities such that each facility attracts a minimum threshold of customers. Drezner and Eiselt (2002) consider a minimum market share threshold to be captured, below which the firm cannot survive and propose the objective of minimizing the probability that revenues fall short of the threshold necessary for survival.

\section{The model.}

The MAXCAP problem seeks the location of a fixed number of stores belonging to a firm in a spatial market where there are other stores belonging to other firms already competing for clients. The objective of the entering firm is to maximize its profits. Whenever the prices charged at the different facilities are equal and there are no location-specific cost differences, the profit-maximizing objective reduces to maximization of sales. 
A customer is an individual or a group with a unique and identifiable location and behavior. Since a customer has a location and issues demand, the term demand point is also used. The expression "point demand" as defined by Plastria (2001) refers to discrete demand concentrated in a finite set of points.

We consider a discrete location space in the sense that there is only a finite list of candidate sites and the market is characterized by point demand.

Each customer feels some attraction towards each of the competing facilities, usually referred as "patronizing behavior". The "attraction function" describes how a customer's attraction, also called utility, towards a facility is obtained.

When we incorporate waiting time in the MAXCAP, customers will patronize a given firm if the sum of the traveling time plus the waiting time at one of its stores is the lowest when compared with other firms' stores.

Let us assume an entering firm (firm A) that wants to locate $p$ new outlets when there are $q$ other outlets from another firm (firm B) already competing at the market place.

In order to solve the problem we consider that the entering firm wants to maximize its market share, that is

$\operatorname{Max}$

$Z=\sum_{i \in I} \sum_{j \in J^{A}} a_{i} X_{i j}$

Where,

$i, I$ index and set of demand points

$j, J$ index and set of potential locations

$J^{A}$ set of firm A's (entrant firm) store locations

$a_{i}$ demand at node $i$

$X_{i j} \quad=1$ if demand point $\mathrm{i}$ patronizes a store at $j$

$=0$ otherwise 
Considering an independent $\mathrm{M} / \mathrm{M} / 1$ queue for each server, the average waiting time at $\mathrm{j}$ is given by:

$$
w_{j}=\frac{\lambda_{j}}{\mu_{j}\left(\mu_{j}-\lambda_{j}\right)}
$$

Where,

$f_{i}$ frequency of persons from demand node $\mathrm{i}$ that will buy the product/service (e.g. persons per hour)

$\mu_{j}$ service rate

As in Marianov and Serra (1998) let us accept the assumption that request for service at each demand point appear according to a Poisson process with intensity $f_{i}$. Each center serves a set of demand points, therefore the requests for service at that center are the union of the requests for service of the nodes in the set. Thus they can be described as a stochastic process equal to the sum of several Poisson processes. The new stochastic process is also a Poisson process, with an intensity $\lambda_{j}$ equal to the sum of the intensities of the processes at the nodes served by the center. This set of nodes will result from the problem's solution. Variables $X_{i j}$ are used in order to rewrite parameter $\lambda_{j}$ :

$$
\lambda_{j}=\sum_{i \in l} f_{i} X_{i j}
$$

If a particular variable $X_{i j}$ is one, meaning that node $i$ is allocated to a center at $j$, the corresponding intensity $f_{i}$ will be included in the computation of $\lambda_{j}$. Let us also assume an exponentially distributed service time, with an average rate of $\mu_{j}$ so that, assuming steady-state each center can be modeled as an M/M/1 queuing system.

Equation (2) can then be rewritten as

$$
w_{j}=\frac{\sum_{i} f_{i} X_{i j}}{\mu_{j}\left(\mu_{j}-\sum_{i} f_{i} X_{i j}\right)}
$$


In order to compute the value of firm A's objective, we need additional information concerning the allocation of demand nodes to the stores defined through variables $X_{i j}$.

Assuming that all customers will patronize the store location that minimizes traveling time plus waiting time, a good estimate for the allocation variables value will result from the minimization of average total time (average traveling time from a demand point to an outlet + average waiting time at a outlet). For each of firm A's potential store locations, and in order to obtain the value of the $X_{i j}$, we solve the following p-median type model:

Min

$$
Z=\lambda_{1} \sum_{i \in I} \sum_{j \in J} a_{i} d_{i j} X_{i j}+\lambda_{2} \sum_{j \in J} \frac{\sum_{i} f_{i} X_{i j}}{\mu_{j}\left(\mu_{j}-\sum_{i} f_{i} X_{i j}\right)}
$$

s.t.

$$
\begin{array}{ll}
\sum_{j \in J} X_{i j}=1 & \forall i \in I \\
\sum_{i \in I} f_{i} X_{i j}<C_{j} & \forall j \in J \\
X_{i j} \in\{0,1\} & \forall i \in I, \forall j \in J
\end{array}
$$

with

$$
\lambda_{1}=\frac{1}{\sum_{i} a_{i}} \quad \text { and } \quad \lambda_{2}=\frac{1}{|J|}
$$

Where the additional notation is the following:

$d_{i j}$ distance from node i to node $j$

$C_{j}$ capacity at store location $j$.

Constraint (6) limits the allocation of one demand point to only one store and constraint (7) fixes the capacity of each store (in order to obtain a finite queue capacity we impose $C_{j}$ to be smaller or equal to $\mu_{j}$ ).

Once the allocations of all the demand points to the stores' location are known it is possible to compute the market share of firm A as given by equation (1). 
Kariv and Hakimi (1979) prove that the p-Median problem is a NP-Hard problem on a general graph. Besides that, notice that the p-median objective is non-linear and that we need to solve a p-median model for each of the possible locations of a firm A store. This explains the important role played by the metaheuristics described in the following section.

\section{Metaheuristics to solve the model.}

\subsection{Description of Metaheuristics .}

Ant Colony Optimization (ACO) introduced by Colorni, Dorigo and Maniezzo (1991) is a cooperative search algorithm inspired by the behavior of real ants. In analogy to the biological example, ACO is based on the indirect communication of a colony of simple agents, called ants, mediated by pheromone trails. The pheromone trails in ACO serve as distributed, numerical information, which the ants use to probabilistically construct solutions to the problem, and which the ants adapt during the execution of the algorithm to reflect their search experience. For a recent description of these metaheuristics, their applications and advances refer to Dorigo and Stützle (2003). For the application to the particular case of an assignment problem, refer to Maniezzo and Colorni (1999) and to Lourenço and Serra (1998).

The problem described can easily be cast into the framework of the ACO metaheuristic. It can be represented by a graph in which the set of components comprises the set of demand points and the set of facility locations. Each assignment will consist of a coupling (i, $j$ ) of demand points and store locations and it corresponds to an ant's walk on the graph.

Lourenço and Serra (1998) present new metaheuristics for the Generalized Assignment Problem. The best result was found using a MAX-MIN Ant System (MMAS), based on an algorithm suggested by Stützle (see as an example Stützle (1998)). Also, Stützle and Hoos (1997) refer the MMAS as one of the most efficient algorithms for the Quadratic Assignment Problem.

The MMAS is an improvement of the more general Ant System metaheuristic, which introduces upper and lower bounds to the values of the pheromone trails, as well as a different initialization of their values. 
The pseudo code for the metaheuristics we used to solve the problem in section 2 is described in Figure 1:

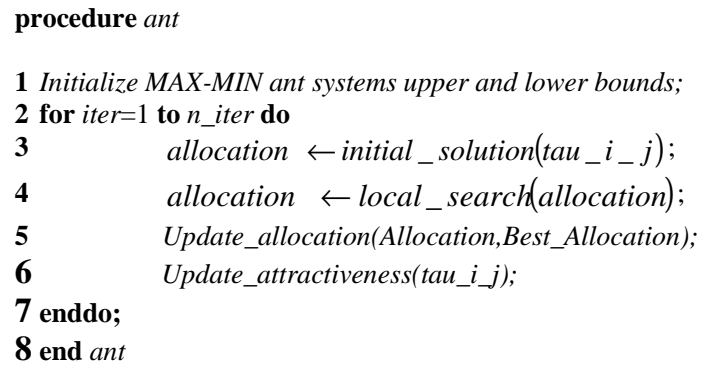

Figure 1: Ant's Algorithm Pseudo Code

In point 1 of the algorithm MMAS upper and lower bounds are initialized. With this purpose we used the following procedure:

1. For each demand point $i$ compute $\tau_{i j}$, the attractiveness to a store located at $\mathrm{j}$ where:

$$
\tau_{i j}=\frac{1}{1+d_{i j}}
$$

The closer it is located, the more attractive the store. At this point of the algorithm it is not possible to compute the waiting time since we do not have information about the allocation of the demand points to the stores.

2. Compute the minimum of $\tau_{\mathrm{ij}}$ and the maximum of $\tau_{\mathrm{ij}}$

3. Compute the lower and upper bounds for the pheromone trails according to the following expressions:

$$
\begin{aligned}
& \tau_{\max }=\max \left(\tau_{i j}\right) \times \text { number of demand points } \\
& \tau_{\min }=0.1 \times \min \left(\tau_{i j}\right)
\end{aligned}
$$

These are the same expressions used in Lourenço and Serra (2000) and they give us initial values for the limits in the MMAS.

At each of the iterations an initial solution is constructed as a function of attractiveness (point 3) and a local search procedure is implemented (point 4). 
The pseudo code for the initial solution procedure is illustrated in figure 2.

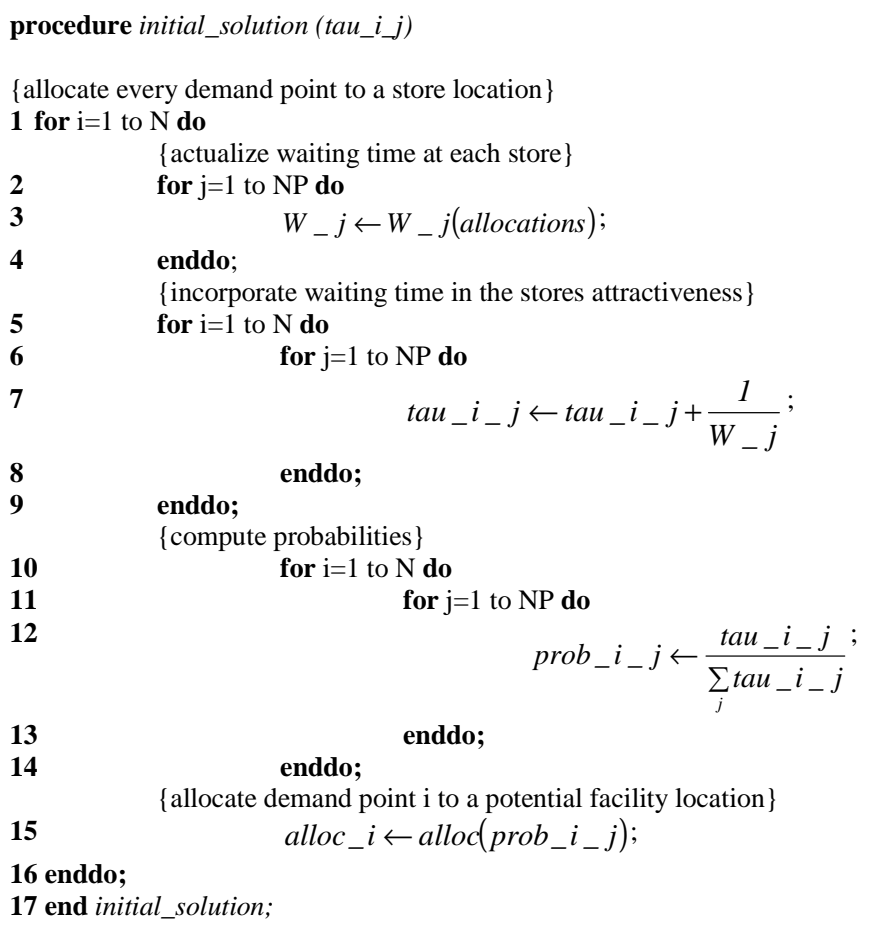

Figure 2: Initial Solution's Algorithm Pseudo Code

Starting with the first demand point in the demand points' list, each demand point will be allocated to a store location according to the following three steps: a) actualize waiting times at the stores, b) actualize stores attractiveness and c) compute new probabilities.

One of the main characteristics of the algorithm is that we are incorporating waiting time at a store location in the attractiveness of that store for all demand points. Attractiveness is inversely correlated with waiting time:

$$
\tau_{i j}= \begin{cases}\tau_{i j}^{\text {new }}+\frac{1}{w_{j}} & \text { if } w_{j} \neq 0 \\ \tau_{i j}^{\text {new }} & \text { otherwise }\end{cases}
$$

Whenever there is a new allocation, waiting time varies and the stores' attractiveness is updated. Since probabilities are positively related to attractiveness, also the probabilities will be updated. 
Each of the demand points are allocated to a potential store location according to the probability rule:

$$
P_{i j}=\frac{\tau_{i j}}{\sum_{j \in J} \tau_{i j}}
$$

where,

$J$ is the set of both firms store locations.

$P_{i j}$ is the probability that one ant will assign demand point $i$ to a potential facility location at $j$.

At this point of the algorithm it this possible to obtain solutions violating constraint (5), i.e. the resulting arrival rate to a store is bigger than the service rate. In order to avoid this solution we opted to penalize the objective with a large value M.

As suggested in Stützle and Hoos (1997) we decided to add a local search phase to the ACO algorithm, in which ants are allowed to improve their solutions. This may improve the performance of the algorithm with respect to quality and convergence speed. The Pseudo Code for the local search phase is illustrated in Figure 3.

The local search phase consisted in the following procedure: de-allocate each demand point $\mathrm{i}$ from potential store location $\mathrm{j}$, and allocate this demand point to each one of the other potential locations. Keeping i new allocation, de-allocate each of the other demand points, one at a time, and check for all possible alternative allocations always computing the respective objective. Whenever the objective improves accept new objective and allocations.

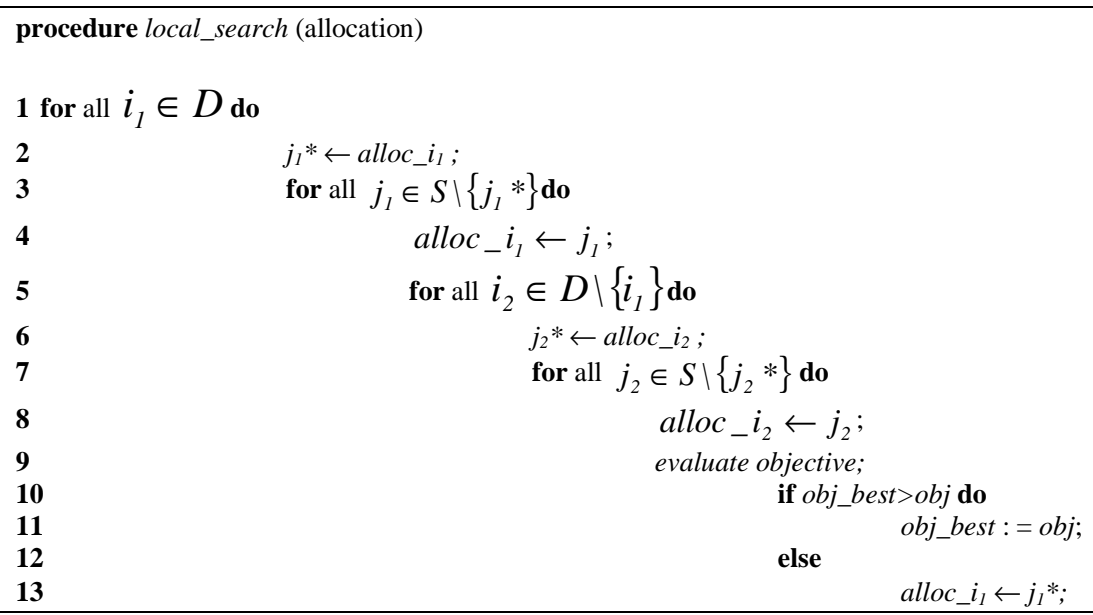




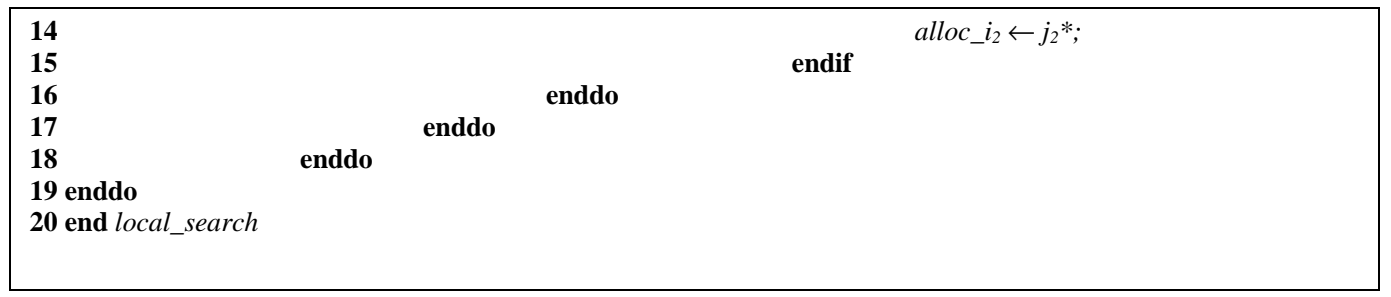

Figure 3: Local Search Algorithm Pseudo Code

In line 6 of the ant procedure (figure 1), the pheromone trails (attractiveness of each demand point to a potential store location) is updated according to the following expression:

$$
\tau_{i j}^{n e w}=\rho \tau_{i j}+\Delta_{i j}
$$

where:

$$
\Delta_{i j}=\left\{\begin{array}{rc}
Q \times \tau_{\max }, & \text { if node } \mathrm{i} \text { is allocated to a facility at } \mathrm{j} \\
0, & \text { otherwise }
\end{array}\right.
$$

and,

$$
Q=\left\{\begin{array}{l}
0.01, \quad \text { if the solution is infeasible } \\
0.05, \quad \text { if the solution is feasible }
\end{array}\right.
$$

Parameter $\rho$ works out as the persistence of the trail; the same is to say that $1-\rho$ gives the evaporation of the pheromone trail. This parameter must be fixed to a value smaller than one to avoid an unlimited accumulation of trace.

In the MMAS pheromone trails must be restricted within upper and lower bounds, i.e.:

$$
\begin{gathered}
\text { if }\left(\tau_{i j}^{\text {new }} \geq \tau_{\max }\right) \\
\tau_{i j}^{\text {new }}=\tau_{\text {max }} \\
\text { if }\left(\tau_{i j}^{\text {new }} \leq \tau_{\text {min }}\right) \\
\tau_{i j}^{\text {new }}=\tau_{\text {min }}
\end{gathered}
$$

For a more detailed exposition of MAX-MIN ant systems see as an example Stützle and Hoos (1998).

\subsection{Analysis of the Metaheuristic performance.}

In order to obtain a measure of the metaheuristics' precision we randomly generated 100 examples and solved the problem of allocating 20 demand points to 3 stores, whose locations 
are known, in order to minimize the sum of average travel time and average waiting time as described through the model in section 3.

For each example we solved the integer problem defined through equations (3)-(6) with a commercial package (LINGO 6) and compared the results with the ones obtained using the metaheuristic suggested in section 2. The results are described in table 3.2.1.

The examples are divided into two groups. The examples defined as "regular examples" consisted of generating both the coordinates as well as the populations from a uniform distribution. The other group of examples results from the use of the procedure described in Cordeau et al (1997). The latter procedure generates instances in which customers tend to be clustered around some fixed centers, as is often the case in real life.

Table 3.2.1: examples with 20 demand points and 3 facilities

\section{Iterations}

Regular examples

$\%$ identical objectives

Average Deviation (\% optimal obj.)

$\%$ identical allocations

Average computing time LINGO

Average computing time Heuristics

Cordeau et al (1997)

$\%$ identical objectives

Average Deviation (\% optimal obj.)

$\%$ identical allocations

Average computing time LINGO

Average computing time Heuristics
25

$78 \%$

$2.23 \%$

$97 \%$

$126.86 \mathrm{~s}$

$3.19 \mathrm{~s}$

$70 \%$

$1.77 \%$

$97 \%$

$16.5 \mathrm{~s}$

$2.34 \mathrm{~s}$
50

$80 \%$

$2.03 \%$

$97 \%$

$126.86 \mathrm{~s}$

$7.28 \mathrm{~s}$

100

$82 \%$

$1.71 \%$

$97 \%$

$126.86 \mathrm{~s}$

$15.75 \mathrm{~s}$

For each one of the examples the metaheurisic was implemented with 25, 50 and 100 iterations.

The results seem to be quite close in terms of identical allocations, which coincides with our initial interest in the metaheuristic. In respect to computing times, the metaheuristic's advantages are clear even for small examples. 


\section{Computational experiments.}

\subsection{Comparison of the results obtained with and without waiting time.}

In the MaxCap model as defined by ReVelle (1986), since waiting time depends on market share and the objective of the firms maximizes market share, there is a tendency for the entrant firm to accumulate large waiting times.

We illustrate this tendency with 30 examples in which firm A wants to locate a new store when there are already two other stores pertaining to firm B operating in the market. In all examples we randomly generated the coordinates and the populations of 20 demand points from a uniform distribution. The coordinates where generated in a $6 \times 6$ square and the populations in the interval $[6000,8000]$. The frequency of people looking for the service by unit of time was fixed at $10 \%$ of the population. Service rate was fixed at 1000 / unit of time. In the examples, we considered that every demand point is also a potential store location.

Let us call the original ReVelle (1986) MaxCap model, model 1, and the model described in section 3, model 2. Results for model 1 were obtained solving the respective integer program in LINGO 6. Results for model 2 were obtained using the metaheuristic defined in section 3 and solving the model for all possible locations for the new firm's store, from which we choose the best one (maximizes market capture).

Table 4.1.1 shows the main results obtained with our experiments. In this table, we see how small the percentage is of our 30 examples from which the use of both models resulted in the same location.

Table 4.1.1: Results from the computational experiments.

Model 1

Model 2

Average waiting time in one outlet

713.8

62.2

Standard deviation for the waiting time in one outlet

867.7

100.6 


\subsection{A numerical example.}

The problem is also illustrated with Swain's (1974) well-known 55-node network. In this example we consider an entrant firm (firm A) that wants to locate a new store when there are already two stores of another firm (firm B) operating in the two demand points' location with the higher populations. Then, we vary the service rate from 0.5 customers per minute to $0.6,0.7$ and 0.8 customers per minute. In Table 4.2.1, we compare the results obtained with model 1 and model 2. Once again results presented as model 1 result from the application of the original formulation of ReVelle's (1986) MaxCap model and the results presented as model 2 result from the application of the model suggested in section 3, evaluating all possible new firm's location.

In all the examples, the arrival rates originating from each of the demand points by unit of time (minute) were fixed at $0.02 \%$ of the respective populations. The Euclidean distances computed from the original coordinates fulfill the distance matrix, measured as traveling time in minutes. In order to simplify the problem the potential store locations were restricted to the 15 demand points with the higher populations.

Table 4.2.1: results for Swain's 55-node network.

\begin{tabular}{|c|c|c|c|c|}
\hline & $\mu=0.5$ & $\mu=0.6$ & $\mu=0.7$ & $\mu=0.8$ \\
\hline \multirow[t]{5}{*}{ Model 1} & Location: 3 & Location: 3 & Location: 3 & Location: 3 \\
\hline & Objective: 1673 & Objective: 1673 & Objective: 1673 & Objective: 1673 \\
\hline & $\mathrm{W}_{3}=5.06$ & $\mathrm{~W}_{3}=2.47$ & $\mathrm{~W}_{3}=1.5$ & $\mathrm{~W}_{3}=1.08$ \\
\hline & $\mathrm{W}_{1}=0.83$ & $\mathrm{~W}_{1}=0.54$ & $\mathrm{~W}_{1}=0.38$ & $\mathrm{~W}_{1}=0.28$ \\
\hline & $\mathrm{W}_{2}=0.10$ & $\mathrm{~W}_{2}=0.07$ & $\mathrm{~W}_{2}=0.05$ & $\mathrm{~W}_{2}=0.04$ \\
\hline \multirow[t]{4}{*}{ Model 2} & Location: 3 & Location: 3 & L Location:3 & Location: 3 \\
\hline & Objective: 1354 & Objective: 1409 & Objective: 1509 & Objective: 1579 \\
\hline & $\mathrm{W}_{3}=2.59$ & $\mathrm{~W}_{3}=1.59$ & $\mathrm{~W}_{3}=1.16$ & $\mathrm{~W}_{3}=0.87$ \\
\hline & $\mathrm{W}_{1}=1.62$ & $\mathrm{~W}_{1}=0.99$ & $\mathrm{~W}_{1}=0.67$ & $\mathrm{~W}_{1}=0.41$ \\
\hline
\end{tabular}




\begin{tabular}{cccc}
\hline \hline $\mathrm{W}_{2}=1.82$ & $\mathrm{~W}_{2}=1.02$ & $\mathrm{~W}_{2}=0.59$ & $\mathrm{~W}_{2}=0.46$ \\
Average traveling & Average traveling & Average traveling & Average traveling \\
Time $: 10.74$ & time $: 10.61$ & time $: 10.59$ & Time $: 10.68$ \\
\hline \hline $\mathrm{W}_{1}$ average waiting time at store $1 ; \mathrm{W}_{2}$ average waiting time at store $2 ; \mathrm{W}_{3}$ average waiting time at store 3 (entrant)
\end{tabular}

$\mathrm{W}_{1}$ average waiting time at store $1 ; \mathrm{W}_{2}$ average waiting time at store $2 ; \mathrm{W}_{3}$ average waiting time at store 3 (entrant)

We can verify how the tendency for the waiting times in the three facility locations becomes similar with increases in the service rate. For lower levels of service rate, the deviation from the waiting time in the new store and the waiting time in the other two stores is clearly greater for model 2. The objectives resulting from both models are different in all the examples. Waiting time has no impact on the objective of model 1 while reducing the objective in model 2 . We give additional information on the average traveling times resulting from model 2.

\section{A Heuristic Concentration algorithm to solve larger problems.}

An obvious limitation of the methodology proposed in the previous sections is the time required to solve larger problems. A possible strategy to diminish this problem is the use of a heuristic concentration algorithm.

Heuristic concentration was developed specifically to deal with larger problems. $\mathrm{HC}$ is a two stage process. Stage 1 involves doing some number (q) of random start runs of an interchange heuristic. A number of these solutions are then subjected to a simple analysis in order to develop the concentration set.

Stage 2 is the construction of a (heuristically derived) good solution or the best solution (by an exact method) from the concentration set. For a detailed description of this methodology, see Rosing and ReVelle (1997) as an example.

A general description of the heuristic concentration algorithm proposed to solve the problem formulated in section 2 consists of the following:

- Stage 1:

1. Find p random initial locations for firm A's stores;

2. Allocate each demand node to its closest store location. Find the demand served by each firm A outlet as well as total firm A market capture. If the utilization factor is bigger than one, set the market capture to zero and go to step 3 . 
3. Choose the first of firm A's outlets from a list of its stores and trade its location to an empty node within the set of potential locations.

4. Find again the demand served by each of firm A's outlets. Compute market capture. If the utilization factor is bigger than one, set the market capture to zero. If market capture has improved, store the new locations. If not, restore the old solution.

5. Repeat steps 3 and 4 until all potential empty locations have been evaluated one at a time for each outlet.

6. If firm A improved its market share to a value greater than in Step 2, go to Step 3 and restart the procedure.

7. When no improvement is achieved for a complete set of one-at-a-time trades, store final solution.

8. Go to Step 1 until a number q of iterations of Stage 1 is met.

- $\quad$ Stage 2:

9. Use all final locations obtained from all starting solutions or use the final locations from the best $\mathrm{k}$ out of the multiple starting solutions in Stage 1 to form the new, reduced set of potential locations (the concentration set - CS).

10. Find $\mathrm{p}$ random initial locations in the CS for firm A's stores;

11. Solve the P-Median model: find the demand served by each of firm A's outlets as well as total market capture of firm A using the ant algorithm described in section 3. If the utilization factor is bigger than one, set the market capture to zero and go to step 9 .

12. Choose the first of firm A's outlets from a list of its stores and trade its location to an empty node within the set of potential locations in the CS.

13. Find again the demand served by each of firm A's outlets using the ant algorithm described in section3. Compute market capture. If the utilization factor is bigger than one, set the market capture to zero. If market capture has improved, store the new locations. If not, restore the old solution. 
14. Repeat steps 3 and 4 until all potential empty locations have been evaluated one at a time for each outlet.

15. If firm A improved its market share to a value greater than in Step 11, go to Step 12 and restart the procedure.

16. When no improvement is achieved for a complete set of one-at-a-time trades, store final solution.

17. Go to Step 10 until a number $p$ of iterations of Stage 2 is met.

In stage one we hope to eliminate some of the potential store locations due to their periphery, increased traveling distances and consequent penalization on the P-Median objective.

We used the heuristic concentration algorithm in order to locate 2 and 3 stores of an entrant firm when there is another firm operating with two stores located in the two demand points with the larger populations.

In our experiments, we compare the solutions obtained using an algorithm that considers all possible combinations for the location of new stores (algorithm 1) with the ones obtained using the above algorithm. For each different combination of number of demand nodes and number of new stores, we randomly generated 10 numerical examples. As in section 4, the examples were generated using the procedure described in Cordeau et al (1997). Coordinates where randomly generated from a uniform distribution on a $6 \times 6$ square, distances are Euclidean, populations were generated from a uniform distribution between 6000 and 8000 and the arrival rates at each demand point were fixed at $10 \%$ of the respective populations. Every demand point is also a potential store location.

Table 5.1: Results from concentration heuristics.

\begin{tabular}{lrrrr}
\hline & 20 nodes & 35 nodes & \\
& 2 stores & 3 stores & 2 stores & 3 stores \\
\hline \hline Algorithm 1 & & & & \\
$\quad$ Average computing time (seconds) & 136.062 & 181.917 & 712.217 & 6263.09 \\
Algorithm 2 & & & & \\
Number of different objectives & 0 & 1 & 0 & 2 \\
Average number of elements in the CS & 12 & 13 & 19 & 23 \\
Average computing time (seconds) & 11.764 & 22.598 & 84.96 & 187.144 \\
Algorithm 3 & & & & 2 \\
Number of different objectives & 0 & 1 & 1 &
\end{tabular}


Given the small size of the examples (20 and 35 nodes) we only considered 100 iterations in stage 1 . The difference between algorithms 2 and 3 consists of the fact that in algorithm 3 , we adopted the procedure of incorporating a new solution in the CS whenever the objective is greater or equal to $90 \%$ of the best objective found at the moment and in the second stage we used complete enumeration for the potential locations in the CS.

Table 5.1 resumes the results obtained with our experiments. In general the HC shows interesting results allowing significant reductions in the problem.

\section{Conclusions.}

The model proposed in this paper seems to be quite useful in the location decisions of new stores for services in which waiting queues are common, as is the case of fast food restaurants, supermarkets or commercial banks.

When the service rate is not large enough relative to the arrival rate which, in turn, results from the market share, waiting time may have a significant impact on the optimal location of a new outlet of an entrant firm.

The metaheuristics we propose in this paper produce results that are close to optimal, offering important savings in computational processing times. 


\section{References}

Carreras, Miquel and Daniel Serra. 1998. "On optimal location with threshold requirements". Socio-Economic Planning Sciences. Vol. 33.pp 91-103.

Colorni, A. , M. Dorigo and V. Maniezzo. (1991). "Distributed Optimization by Ant Colonies". Proceedings of ECAL91- European Conference on Artificial Life. Elsevier Publishing.pp 134142.

Cordeau, J. F., M. Gendreau and G. Laporte. 1997. "A tabu search algorithm for periodic and multi-depot vehicle routing problems". Networks. Vol. 30. 105-119.

Dorigo, Marco and Thomas Stützle. 2003. "The ant colony optimization metaheuristics: algorithms, applications and advances" in Handbook of Metaheuristics. Edited by Fred Glover and Gary Kochenberger. Kluwer Academic Publishers.

Drezner, Tammy and H.A. Eiselt. 2002. "Consumers in competitive location models". In Facility Location: Applications and Theory. Edited by Zvi Drezner and H.W. Hemacher. Springer Verlag.

Drezner, Tammy. 1994. "Locating a single new facility among existing, unequally attractive facilities". Journal of Regional Science. Vol. 34. N. 2. pp 237-252.

Eiselt, H.A. and Gilbert Laporte. 1989. "The maximum capture problem in a weighted network". Journal of Regional Science. Vol. 29. N. 3.pp 433-439.

Hakimi, S.L. 1983. "On locating new facilities in a competitive environment". European Journal of Operational Research. Vol. 12. 29-35.

Kariv, O and S.L. Hakimi. 1979. "An algorithm Approach to Network Location Problems, Part II: the P-median". SIAM journal of Applied Mathematics. Vol. 37. 539-569.

Kohlberg, Elan. 1983. "Equilibrium store locations when consumers minimize travel time plus waiting time". Economics Letters. Vol. 11.pp 211-216.

Lourenço, H. and Daniel Serra. 1998. "Adaptive approach heuristics for the generalized assignment problem". Economics Working Paper 288. Universitat Pompeu Fabra.

Maniezzo, Vittorio and Alberto Colorni. 1999. "The ant system applied to the quadratic assignment problem". IEEE Transactions on knowledge and data engineering. Vol. 11. N.5.

ReVelle, Charles. 1986."The maximum capture or sphere of influence location problem: Hotelling revised on a network". Journal of Regional Science. Vol. 26. N. 2.

Rosing, K.E. and Charles ReVelle. 1997. "Heuristic Concentration: Two Stage Solution Construction". European Journal of Operational Research. Vol. 97.pp 75-86.

Plastria, Frank. 2001. "Static competitive facility location: an overview of optimization approaches". European Journal of Operational Research. .Vol. 129. pp 461-470.

Serra, Daniel and Charles ReVelle. 1993. "The pq-median problem: location and districting of hierarchical facilities”. Location Science. Vol. 1. N. 4.pp 299-312.

Serra, Daniel and Charles ReVelle. 1994. "Market capture by two competitors: the preemptive 
location problem”. Journal of Regional Science. Vol. 34. N. 4.pp 549-561.

Serra, Daniel and Charles ReVelle. 1995. "Competitive location in discrete space". In Facility Location: Applications and Methods. Edited by Zvi Drezner. Springer Verlag.

Serra, Daniel, Charles ReVelle and Ken Rosing. 1999. "Surviving in a competitive spatial market: the threshold capture model". Journal of Regional Science. Vol. 39. N.4.pp 637-652.

Serra, Daniel, H. A. Eiselt, Gilbert Laporte and Charles ReVelle. 1999. "Market capture models under various customer choice rules" Environment and Planning B. Vol. 26. N. 5.pp 741-750.

Serra, Daniel, S. Ratick and Charles ReVelle. 1996. "The maximum capture problem with uncertainty". Environment and Planning B. Vol. 23. N. 4.pp 49-59.

Stützle and Hoos .1997. "MAX-MIN ant system for Quadratic Assignment Problems". TH Darmstadt, FB. Informatik Darmstadt. Germany.

Stützle and Hoos .1998. "Improvements on the Ant System: Introducing MAX-MIN Ant System ". in Artificial Neural Networks and Genetic Algorithms. Edited by G. D. Smith and N.C. Steele, R.A., pages 245-249.

Stützle, Thomas. 1998. "An ant approach for the flow shop problem". TH Darmstadt, FB. Informatik Darmstadt. Germany.

Swain, Ralph. 1974. "A parametric decomposition algorithm for the solution of uncapacitated location problem" Management Science. Vol. 21. 189-198. 